Conclusion Bleeding per rectum is an infrequent presentation to the PED. The most common diagnoses are benign and may be managed without need of invasive investigation, admission or outpatient follow up. A high index of suspicion remains necessary to identify infrequent but serious pathology.

\section{G225(P) THE INCIDENCE AND OUTCOMES OF PNEUMOTHORAX IN NEONATES IN THE $21^{\text {ST }}$ CENTURY}

1,2 L Miall, ${ }^{1,2} \mathrm{H}$ Shore, ${ }^{2} \mathrm{~S}$ Salar, ${ }^{2} \mathrm{G}$ Dunbavand. ${ }^{1}$ Neonatal Intensive Care Unit, Leeds Teaching Hospitals Trust, National Health Service, Leeds, UK; ${ }^{2}$ Extended Student Research Evaluation Project, University of Leeds Medical School, Leeds, UK

\subsection{6/archdischild-2020-rcpch.193}

Introduction A pneumothorax is most common in the neonatal period. There are many risk factors associated with its development including meconium aspiration syndrome, respiratory distress syndrome, pulmonary hypoplasia and mechanical ventilation (MV). Definitive management includes: expectant therapy, thoracentesis or insertion of a chest drain.

Aims To describe the incidence, aetiology and outcomes of pneumothorax in a tertiary neonatal unit to determine the risk factors with the most clinical relevance to prognosis.

Methods This study was a retrospective cross-sectional analysis of 9446 patients admitted to the NICU between August 2010 and December 2018, of which 178 met the inclusion criteria. There were 80,000 live births during this 8 -year period. The data was taken from the Badger.net neonatal patient database, local electronic record and local radiology imaging archive. Analysis used Microsoft Excel and SPSS.

Results The incidence of pneumothorax was 2 per 1000 live births (0.22\%) and 19 per 1000 NICU admissions (1.9\%). The overall mortality was $20 \%$. The median gestation was 37 +5 and the mean birth weight was $2475 \mathrm{~g}$. Males accounted for $63 \%(p=1.43)$ and females $37 \%(p=1.87)$. Of the total, $61 \%$ had a unilateral right-sided pneumothorax while unilateral left-sided pneumothorax accounted for 11\%. Birth weight was a significant risk factor for mortality $(p=0.002$, OR 0.998 CI $0.997-0.999)$. Of the $44 \%$ of neonates that had expectant management only, the mortality before discharge was $5 \%$. Of the $56 \%$ that had a chest drain inserted, pre-discharge mortality was $33 \%$. Of the term infants, 24 neonates received expectant management and had no $\mathrm{MV}$ prior to or after diagnosis. The survival rate in this group was $100 \%$ and the recurrence rate was $0 \%$. Amongst preterm neonates, there was a $28 \%$ chance of recurrence compared to $10 \%$ in term infants.

Conclusion The overall pre-discharge mortality in the cases was $20 \%$ with preterm neonates being more likely to have a recurrent pneumothorax and a higher rate of mortality. As birth weight decreases, mortality increases. Term neonates without an acute requirement for respiratory support can often be safely managed with expectant therapy.

\section{G226(P) IT WILL STICK LIKE GLUE: IMPROVING THE MANAGEMENT OF SIMPLE LACERATIONS IN A\&E}

H Jones, S Baskind, W Niven, N MacLeod, N George, J Bowen. AandE, Homerton University Hospital, London, UK

10.1136/archdischild-2020-rcpch. 194
Background Lacerations are the 6th most common presentation to A\&E in the UK. These vary from complex wounds requiring specialist review to those that can be repaired with steri-strips or glue. Most wounds are simple enough to be repaired by the nurse who first assessed the child. However, local practice in Children's Emergency Area (CEA) has been for every wound to be reviewed by a doctor. Consequences include long waiting times and crowded waiting rooms.

Aim Decrease time spent in CEA by children with 'simple lacerations' to 90 minutes by September 2019.

Methodology PDSA Cycle 1: A Standard Operating Procedure (SOP), designed by our multi-disciplinary team, was implemented on 18th August 2019. This sets a standard management pathway for children presenting to CEA with lacerations, and allows a nurse-led-discharge for 'simple lacerations' which do not meet exclusion criteria.

Average encounter times (AET's) were recorded on a Run Chart for the first six children presenting to CEA with 'simple lacerations', each week between March-October 2019. Prior to implementation, only AET's for lacerations which could have been managed by nurses were included. After implementation, AET's reflect the first six patients managed by either nurses or doctors.

We compared AET's between 31st March-18th August prior to implementation, to AET's between 18thAugust-15th October after implementation.

PDSA Cycle 2: Senior CEA nursing staff provided teaching to improve confidence and adherence to the SOP.

Results The average AET prior to implementation was 192 minutes. After implementation, average AET reduced to 117 minutes.

Since implementation, $52 \%$ were managed by nurses, with an average AET of 80 . In the same time period, average AET for those managed by doctors was 163

Conclusion Using the SOP resulted in significant improvement of overall AET's in CEA. AET's for nurse-led-discharges were lower than for doctor-led-discharges.

Lower AET's result in lesser exposure to pathogens in the waiting room and allow doctors more time with acutely unwell children. We hope the pathway could serve as a model for other CEA's across the UK.

With evidence that AET's are shorter for nurse-led-discharges, our next PDSA cycle will focus on increasing number managed by nurses. We will monitor balancing measures: returning patients, and patient satisfaction.

\section{G227(P) LIQUID DETERGENT CAPSULES ARE THE COMMONEST CAUSE OF ACCIDENTAL POISONING IN CHILDREN LESS THAN 5 YEARS-OLD PRESENTING TO TWO UK EMERGENCY DEPARTMENTS}

${ }^{1} \mathrm{MBKC}$ Dayasiri, ${ }^{1} \mathrm{~J}$ Peppiatt, ${ }^{2} \mathrm{C}$ Bird. 'Department of Paediatrics, Oxford University Hospital, Oxford, UKi '2Emergency Department, Birmingham Children's Hospital, Birmingham, UK

\subsection{6/archdischild-2020-rcpch.195}

Aims Despite a global campaign on the dangers presented by liquid detergent capsules (LDC) - which look like sweets to a toddler - young children continue to present to emergency departments with LDC ingestion. We aimed to determine how often LDCs, or 'liquitabs', were the cause of children 
presenting with accidental poisoning to our two emergency departments.

Methods We performed a retrospective analysis of all children less than 5 years old and presenting between July 2016 and July 2018 with an accidental poisoning to the emergency departments at the John Radcliffe Hospital, Oxford, and the Horton Hospital, Banbury. Children with either deliberate, food or unknown poisoning were excluded. As an audit, the study did not require ethical approval.

Results Among 214 children with accidental poisoning (median age of 2 years, $52.3 \%$ male), LDCs were the commonest cause (27 cases, 12.6\%), followed by paracetamol (15, 7\%) and carbon monoxide (10,4.7\%). There were 103 different substances recorded, with personal care products forming the largest group $(110,51.4 \%)$ followed by medications (57, $26.6 \%)$.

Most children were asymptomatic $(173,80.8 \%)$. The commonest symptom was vomiting $(22,10.3 \%)$. A quarter had an ECG performed $(47,22 \%)$ and $31(14.5 \%)$ children had a blood test. All children were discharged from the emergency department after a period of observation.

Conclusion LDCs were the commonest cause of accidental poisoning in young children presenting to our emergency departments. Healthcare workers should engage with industry to make packaging of LDCs safer to prevent continued presentations by young children with LDC poisoning.

\section{G228(P) ABSTRACT WITHDRAWN}

\section{G229(P) USING SIM TO ADDRESS THE NEEDS OF THE 'FORGOTTEN TRIBE'}

S Mullen, P Martin, L Loughins, N Wetherup. PED, RBSCH, Belfast, UK

\subsection{6/archdischild-2020-rcpch.196}

Aims Adolescences make up approximately 19\% of the UK population with treatment received split between paediatric and adult services. Research has noted that adolescent attend $\mathrm{ED}$, often as a first preference for illness/injury. The aim was to enhance local knowledge and skills regarding adolescent care with an emphasis placed on emergency presentations.

Methods We developed a one-day teaching programme consisting of mixed teaching modalities (lecture, case-based discussions and simulations). The learning objectives were tailored to the curriculum for paediatric and emergency medicine trainees with input from local experts in adolescent health. A multi-disciplinary faculty facilitated including clinicians from paediatric emergency medicine, emergency medicine, paediatrics, CAMHS (Child and adolescent mental health services) and a speciality organ donation nurse.

A total of eight high fidelity simulations were developed and delivered by faculty covering adolescent trauma, DKA, intoxication, the aggressive adolescent, HEEADSSS (psychosocial) assessment, confidentiality, capacity and utilising the Mental Health Act. Amateur actors were employed to play the roles of patients/parents to enhance fidelity.

Results There were 36 participants who attended with $86 \%$ $(\mathrm{n}=31)$ completing the pre-course questionnaire and $95 \%$ $(n=34)$ completing the post course questionnaire. An increase in confidence and knowledge was seen across all domains for all trainees, with an average overall improvement of $30 \%$. The greatest increase in confidence was noted in managing an aggressive adolescent with verbal de-escalation $(55.8 \%$ vs $82.1 \%$ pre and post), managing an adolescent refusing treatment $(43.9 \%$ vs $80.6 \%$ pre and post) and psychosocial evaluation using the HEEADSSS assessment tool $(26.2 \%$ vs $81.8 \%$ pre and post). Feedback was universally positive. Comments included 'Relevant and interactive, learned so much' and 'fantastic course. Covered areas I didn't know I was lacking in.' Delayed feedback at 3 months showed sustained confidence across all areas and evidence of new skills learned being used in clinical practice - in particular use of the HEEADSSS assessment. All candidates would recommend the course to colleagues.

Conclusion This project is an excellent showcase of how we can bring a group of clinicians from various different backgrounds together to drive improvement for a vulnerable group of patients.

\section{G230(P) CAN UTILISING OUT-PATIENTS SPACE IMPROVE SERVICES OFFERED IN A PED?}

S Mullen, T Oman, E Dalzell, A Fitzsimons, B Bartholome, H Jahn. PED, RBHSC, Belfast, UK

\subsection{6/archdischild-2020-rcpch.197}

Aims The PED that has seen a 10000 increase in attendances over the last 5 years with no increase in space to accommodate this. This led to issues with patient flow, poor morale and increase in waiting times. Our aim was to utilise outpatient clinic space to run a minor illness and injury area for emergency department attendances.

Methods This initiative utilised fracture out-patient clinic and staffing with middle grade paediatric or emergency staff. In addition, this service was staffed by a band 3 receptionist, an enhanced nurse practitioner and a band 5 nurse. The clinic runs on the three peak days of the week (Monday, Tuesday and Friday) between 17:30 to 22:30.

Results 404 patients were seen over 31-days, averaging 13 patients per day. A survey was completed by 52 patients/ parents. 98\% reported that this was an appropriate area to be seen with another $98 \%$ stating that the Winter pressures clinic was beneficial. They rated there experience as 4.1 out of 5 (mean).

Comparison between this year (2018/9) and previous (2017/8) demonstrated a reduction in average time in ED of 11 minutes per patient for all category four and five patients despite significantly higher numbers.

The comments from parents supported the use of this initiative; ' $\mathrm{A}+E$ was a nightmare, overcrowded and not a nice environment. Minor unit is quiet and less stressful for the baby and parent', 'Getting moved to the other emergency department today was for the better'. Staff have been very supportive of this service. Comments acknowledged the ability to provide a more efficient service to those of a lower triage category. The service was also found to be beneficial to the higher acuity patients in the ED allowing them to be allocated to a treatment room quicker and prioritising staff to see them in a more efficient and timely manner.

Conclusion The use of an out-patient clinical space is an appropriate area to manage minor illness and injury in the emergency department. It has improved flow, reduced waiting times and is acceptable area for patients and staff. 